E. Stephan 1 - St. Kistryn • I. Skwira-Chalot - I. Ciepał •

B. Kłos • A. Kozela · W. Parol • A. Rusnok • A. Wilczek •

J. Zejma

\title{
Dynamics of Three-Nucleon System Studied in Deuteron-Proton Breakup Experiments
}

\author{
New Set of Invariant Coordinates
}

Received: 19 September 2016 / Accepted: 7 December 2016 / Published online: 11 January 2017

(C) The Author(s) 2017. This article is published with open access at Springerlink.com

\begin{abstract}
Systems composed of three nucleons have been a subject of precise experimental studies for many years. Recently, the database of observables for the deuteron breakup in collision with protons has been significantly extended at intermediate energies. In this region the comparison with exact theoretical calculations is possible, while the sensitivity to various aspects of the interaction, in particular to the subtle effects of the dynamics beyond the pairwise nucleon-nucleon force, is significant. The Coulomb interaction and relativistic effects show also their influence on the observables of the breakup reaction. All these effects vary with energy and appear with different strength in certain observables and phase-space regions, which calls for systematic investigations of a possibly rich set of observables determined in a wide range of energies. Moreover, a systematic comparison with theoretical predictions performed in coordinates related to the system dynamics in a possibly direct way is of importance. The examples of existing experimental data for the breakup reaction are briefly presented and the amenability of a set of invariant coordinates for that type of analysis is discussed.
\end{abstract}

\section{Introduction}

The understanding of the structure and dynamics of nuclei as systems of interacting protons and neutrons is one of the major goals of nuclear physics. The nucleon-nucleon potential is the leading part of the nuclear interaction and should be sufficient to describe basic properties of nuclei and main trends in observables for systems of few (and many) nucleons, if exact calculations are feasible. However, since the internal structure of nucleons is neglected, the question arises, how the suppressed degrees of freedom influence the observables in any system consisting of more than 2 nucleons. Such additional dynamics, called three-nucleon force (3NF), cannot be reduced to pairwise forces. It arises in the meson-exchange picture as an intermediate excitation of

This article belongs to the Topical Collection "30th anniversary of Few-Body Systems".

This work was partially supported by Polish National Science Center from Grant DEC-2012/05/B/ST2/02556 and by the European Commission within the Seventh Framework Programme through IA-ENSAR (Contract No. RII3-CT-2010-262010).

E. Stephan $(\varangle) \cdot$ B. Kłos · A. Rusnok · A. Wilczek

Institute of Physics, University of Silesia, 40007 Katowice, Poland

E-mail: elzbieta.stephan@us.edu.pl

St. Kistryn · J. Zejma

Institute of Physics, Jagiellonian University, 30348 Kraków, Poland

I. Skwira-Chalot

Faculty of Physics, University of Warsaw, 02093 Warsaw, Poland

I. Ciepał · A. Kozela $\cdot$ W. Parol

Institute of Nuclear Physics, Polish Academy of Sciences, 31342 Kraków, Poland 
a nucleon to a $\Delta$ isobar, or it appears fully naturally in Chiral Effective Field Theory at a certain order [1]. In the first case, 3NF models, like TM99 [2] or Urbana IX [3] forces, are combined with a given realistic nucleon-nucleon $(2 \mathrm{~N})$ potential. Alternatively, the $\Delta$ isobar is included explicitly and the coupled-channel framework is applied [4]. Three-nucleon systems, as amenable to accurate ab-initio calculations, represent an excellent testing ground for $2 \mathrm{~N}+3 \mathrm{NF}$ interactions, constructed in any of the ways mentioned here.

The importance of $3 \mathrm{NF}$ contributions to the dynamics of systems of more than two nucleons was first established in binding energies of few-nucleon states [5-8]. Further verification of the role of the 3NF has been carried out on the basis of scattering experiments: the measurements of observables for elastic nucleondeuteron scattering and for the breakup of a deuteron in its collision with a nucleon. The extensive discussions of the present status of our understanding of the $3 \mathrm{~N}$ system dynamics, based on modern calculations and many precise and rich data sets, can be found in recent reviews [9-11]. The 3NF turned out to be very important for improving the description of the cross section for nucleon-deuteron elastic scattering. At beam energies above $100 \mathrm{MeV}$ per nucleon certain discrepancies between data and calculations still persist, though significantly reduced as compared to predictions based on purely $2 \mathrm{~N}$ potentials (cf. [12] and references therein). On the other hand, the precise experimental data demonstrate both the successes and the difficulties of the current models in describing analyzing powers, spin-transfer and spin-correlation coefficients for $N d$ elastic scattering.

Deuteron breakup in collision with a proton is another source of information on the three-nucleon system dynamics. When studied with a detector covering a big part of the phase space, it provides extensive information, and the effects of various dynamical ingredients can be studied as a function of a set of kinematic variables. Such an approach was applied in a series of experiments carried out at KVI, which comprised the studies of the ${ }^{1} \mathrm{H}(\mathbf{d}, \mathrm{pp}) \mathrm{n}$ and ${ }^{2} \mathrm{H}(\mathbf{p}, \mathrm{pp}) \mathrm{n}$ reactions with deuteron beams of energies 100,130 and $160 \mathrm{MeV}(50,65$, $80 \mathrm{MeV} /$ nucleon) and proton beams with energies of 135 and $190 \mathrm{MeV}$. The detection systems of large angular acceptance, SALAD and BINA [11-13], were employed. Regarding differential cross sections, an experiment using the $4 \pi$ WASA detector and deuteron beams of energies from 170 to $200 \mathrm{MeV} /$ nucleon has recently been performed at the COSY ring of FZ-Jülich, while the investigations at lower (proton) beam energies between 108 and $160 \mathrm{MeV}$ are currenly being carried out with the use of the BINA detector at the newly opened Cyclotron Center Bronowice (Cracow, Poland).

To a large extent, the general conclusions about the role of $3 \mathrm{NF}$ contributions for the description of the cross section for the breakup reaction are so far similar to those for elastic scattering. The significance of the $3 \mathrm{NF}$ for a correct description of the differential cross section has been confirmed [14,15]. On the other hand, certain discrepancies are observed for the tensor analyzing powers of the breakup reaction at the same beam energies, even if (or when) the 3NF is included in the calculations [16]. The experimental studies of the ${ }^{2} \mathrm{H}(\mathbf{p}, \mathrm{pp}) \mathrm{n}$ reaction at 135 and $190 \mathrm{MeV}[17,18]$ show a large (and growing with beam energy) discrepancy between the measured data and the theoretical predictions for the vector analyzing power $A_{y}^{p}$. It is located at small relative azimuthal angles of the two breakup protons, and even increases with the three-nucleon force included. The predicted relativistic effects do not explain this behaviour. These facts confirm a problem with the description of spin observables in $3 \mathrm{~N}$ systems. The precise data sets for polarization observables of the ${ }^{1} \mathrm{H}(\mathbf{d}, \mathrm{pp})$ n reaction at the beam energy of $270 \mathrm{MeV}$, collected at IUCF [19] and at RIKEN [20], also showed "a mixed picture" in the sector of spin observables. For example, $A_{y}^{d}$ obtained in several angular configurations is described properly by the pure $2 \mathrm{~N}$ force predictions, while the inclusion of the $3 \mathrm{NFs}$ leads to the deterioration of the agreement [20]. Such a behaviour is opposite to the one observed in elastic scattering at $270 \mathrm{MeV}$.

Elastic scattering is practically insensitive to the Coulomb interaction [21] and relativistic effects [22] over a wide range of energies. In contrast, the breakup reaction reveals, due to the variety of configurations of its final state, sensitivity to both of these effects, particularly enhanced at specific kinematics. In view of interplays of various effects in the breakup reaction, it is of great importance to compare experimental data to calculations which include all the dynamical ingredients. Significant progress in this respect has been achieved in recent years, and currently the calculations combining $3 \mathrm{NF}$ and Coulomb interactions are available [23] as well as relativistic calculations including 3NFs [22]. Coulomb effects turned out to be surprisingly large [15,24]. Therefore, an experiment probing the part of the phase space that is particularly sensitive to the Coulomb interaction was conducted at FZ-Jülich. A deuteron beam of $130 \mathrm{MeV}$ and the GeWall detector [25] covering very forward angles were used in these studies. A strong influence of Coulomb effects and the general success of the theoretical calculations incorporating this long range interaction were confirmed [26]. The importance of relativistic calculations for describing the breakup reaction cross section has been shown at energies as low as $65 \mathrm{MeV}$ ([27] using data from [28,29]). The calculations will be further tested by comparing with results of the breakup cross section measurements performed with the WASA detector at COSY [31] in the energy range where predicted relativistic effects exceed 30\% [30]. 


\section{Analysis Using Invariant Coordinates}

The interplay of all dynamical effects in the breakup process requires a large database covering a wide range of energies, in order to verify the theoretical calculations. An optimal choice of kinematic coordinates is important for a systematic survey of such a database. In spite of the equivalence of many possible choices, the coordinates with possibly close relation to the dynamics of the process could be of particular interest. The relative momentum of a pair of outgoing particles is one example of this type, giving direct insight into the Final State Interaction (FSI) of this pair. For a 3-nucleon final state it is quite natural to define two such variables: for the pair of identical nucleons ( $p p$ in the case of $p d$ breakup process) and for the $n p$ pair. Low relative momentum of the proton-neutron pair corresponds to configurations "similar" to elastic $d p$ scattering, while for the pair of protons large effects of the Coulomb interaction are expected at the minimum value of such a variable. The motivation for the choice of two other coordinates is based on the important role of the four-momentum transfer in the two-body reaction dynamics. The differential cross section of elastic proton-deuteron scattering at small polar angles in the center-of-mass system (low momentum transfer) is dominated by the direct term in the two-nucleon potential and is rather well described by the calculations using only two-nucleon forces. At backward angles (large momentum transfer), the nucleon exchange term in the two-nucleon potential is responsible for the increase of the cross section. In these two regions the effects of three-nucleon forces are predicted to be mimimal. At intermediate angles, where the cross section has a minimum, it is expected that the effects of three-nucleon forces will be significant. In breakup, for the threebody exit channel, the 4-momentum transfer can be defined separately for individual nucleons. Intuitively, the less the momentum of the nucleon changes during the process, the less the nucleon is "involved" in the interaction, at the zero limit playing mainly the role of a spectator, which corresponds to Quasi-Free Scattering (QFS) of the other two nucleons. That is the reason why the so-called Chew-Low plot, combining invariant mass with 4-momentum transfer, was once used for breakup analyses [32,33] to identify events originating from quasi-elastic scattering in particular.

The coordinates can be constructed in analogy to the Mandelstam invariants. Denoting the four momenta in the exit channel by $p_{p}^{(1)}, p_{p}^{(2)}$ (for two protons) and $p_{n}$ (for a neutron), and in the entrance channel by $p_{p}$ for a proton and $p_{d} / 2$ for any of the nucleons bound in a deuteron, we propose the following set of invariants (for further discussion see [11]):

$-s_{p p}=\left(p_{p}^{(1)}+p_{p}^{(2)}\right)^{2}$ for a pair of protons in the exit channel,

$-s_{p n} \equiv s_{p^{(1)} n}=\left(p_{p}^{(1)}+p_{n}\right)^{2}$ for a proton-neutron pair - arbitrarily the pair with the lower $s_{p n}$ value can be chosen,

$-t_{n}=\left(p_{d} / 2-p_{n}\right)^{2}$, corresponding to the 4-momentum transfer from a bound neutron in the entrance channel to a free neutron in the exit channel,

- $t_{p}=\left(p_{p}-p_{p}^{(2)}\right)^{2}$, corresponding to the 4-momentum transfer from an unbound proton in the entrance channel to one of the two protons in the exit channel - arbitrarily, we choose the proton, which was not used in the calculation of $s_{p n}$.

One can easily recognize that the so-called star configurations are characterized by $s_{p p}=s_{p n}$ (with its value dependent on beam energy), while variation of the angle between the star plane and beam directions, $\alpha$, can be translated to variation of $t_{n}$. This is only one out of many examples of a simple and clear description given in terms of the proposed coordinates, apart from the already mentioned FSI and QFS.

For convenience, the 4-momenta based coordinates can be redefined as energies, corresponding to the energy of the relative motion of two nucleons ( $p p, p n$ pairs), $E_{r e l}^{p p}, E_{r e l}^{p n}$, and the kinetic energy "transferred" to a particle:

$$
\begin{aligned}
E_{r e l}^{p p} & =\sqrt{s_{p p}}-2 m_{p}, \\
E_{r e l}^{p n} & =\sqrt{s_{p n}}-m_{p}-m_{n}, \\
E_{t r}^{p} & =\frac{-t_{p}}{2 m_{p}}, \\
E_{t r}^{n} & =\frac{-t_{n}}{2 m_{n}} .
\end{aligned}
$$

The full dynamical information is maintained only on the 4-dimensional surface, while the integration over any number of coordinates leads to obscuring of parts of the information. However, if an effect is 

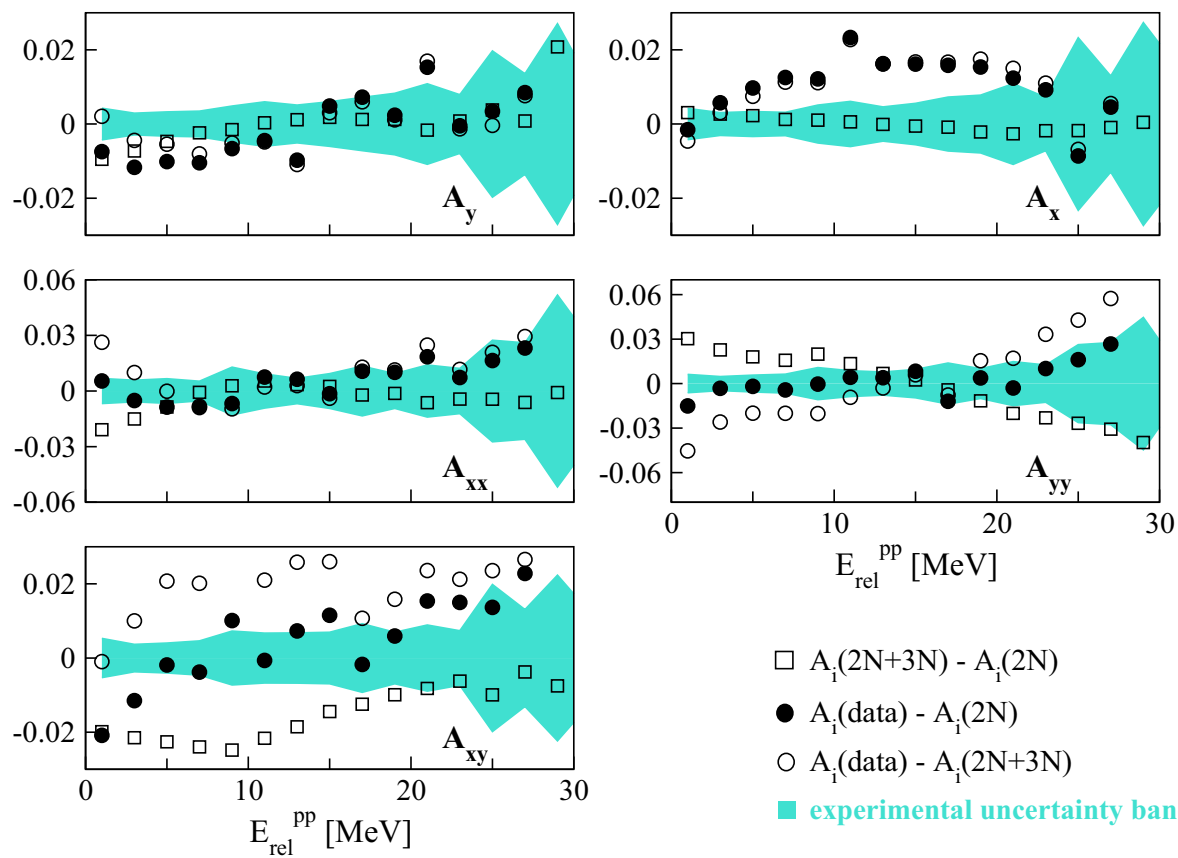

$$
\begin{aligned}
& \square \mathrm{A}_{\mathrm{i}}(2 \mathrm{~N}+3 \mathrm{~N})-\mathrm{A}_{\mathrm{i}}(2 \mathrm{~N}) \\
& \text { - } \mathrm{A}_{\mathrm{i}}(\text { data })-\mathrm{A}_{\mathrm{i}}(2 \mathrm{~N}) \\
& O \mathrm{~A}_{\mathrm{i}}(\text { data })-\mathrm{A}_{\mathrm{i}}(2 \mathrm{~N}+3 \mathrm{~N}) \\
& \text { experimental uncertainty band }
\end{aligned}
$$

Fig. 1 Net effects of the $3 \mathrm{NF}$ on vector and tensor analyzing powers of the $d p$ breakup reaction measured at the deuteron beam energy of $130 \mathrm{MeV}$. Open squares represent the predicted effects, i.e. the difference between the results of calculations including the TM99 $3 \mathrm{NF}$ and those based on pure $2 \mathrm{~N}$ potentials. Circles represent the difference between the measured and calculated observables, as explained in the legend, with the experimental uncertainty shown as bands. Theoretical calculations by Witała et al. For details see text

strongly correlated with a specific coordinate, it should not be smeared out by the integration and it stays clearly visible even in a one-dimensional spectrum. Such an analysis, performed in terms of $E_{r e l}^{p p}$, turned out to be a very useful tool for tracing $3 \mathrm{NFs}$ and, in particular, Coulomb effects in the breakup cross section at $130 \mathrm{MeV}$ (65 MeV/nucleon) [11]. The latter is consistent with the intuitive understanding of the relation between Coulomb effects and proton-proton FSI.

A similar analysis for vector and tensor analysing powers is presented here (a fraction of the results has already been discussed in [34]). The theoretical values were calculated for each vector and tensor analyzing power $A_{i}$ at each experimental point, analysed conventionally on a grid of $\xi=\left(\theta_{1}, \theta_{2}, \phi_{12}, S\right)$ variables [11]. Then both data and theoretical predictions were sorted into $E_{r e l}^{p p}$ bins of $2 \mathrm{MeV}$. The predictions based on a set of two-nucleon ( $2 \mathrm{~N}$ ) realistic potentials (Argonne V18, Nijmegen I, II and CD-Bonn) were compared with the calculations including the TM99 $3 \mathrm{NF}(2 \mathrm{~N}+3 \mathrm{~N})$ with the aim to analyse $3 \mathrm{NF}$ effects. The predictions obtained with the $2 \mathrm{~N}$ potentials alone were considered together creating a band. The center of this band was then used for further comparisons with the data. The $2 \mathrm{~N}+3 \mathrm{~N}$ calculations were treated in a similar way. Since the bands are relatively narrow (as compared to the effects under study) such an approach is justified. In order to magnify the effects, the theoretical predictions have been subtracted from the data. In addition, the difference between the two types of predictions has been calculated, see Fig. 1. Squares on the zero level represent the situation when the predicted $3 \mathrm{NF}$ effect is negligible. This is the case for $A_{x}, A_{y}$ and $A_{x x}$. The predicted effects of the $3 \mathrm{NF}$ for $A_{y y}$ and $A_{x y}$ are sizeable and they exceed the experimental uncertainties, therefore the data are very suitable for testing them. For $A_{y y}$ one can observe a systematic change of the predicted effect with $E_{r e l}^{p p}$, signalling the importance of that coordinate in the description of the system dynamics. Full and open circles represent the difference between data and theory: the theoretical description is validated if the symbols are contained within the uncertainty bands. The most significant discrepancy is observed for $A_{y y}$ and $A_{x y}$ when the calculations with 3NFs (open circles) are applied. Apparently, the predicted effect is not confirmed with the data set.

It can be argued that Coulomb effects (not included in the calculations used in the above comparisons) may be the reason of the discrepancies. Therefore, the data were compared to calculations based on the Argonne V18 (AV18) potential combined with Urbana IX 3NF (UIX), with (AV18 + UIX + C) and without Coulomb interaction (AV18+UIX) [23] in order to verify that hypothesis. As in the previous case, the theoretical 

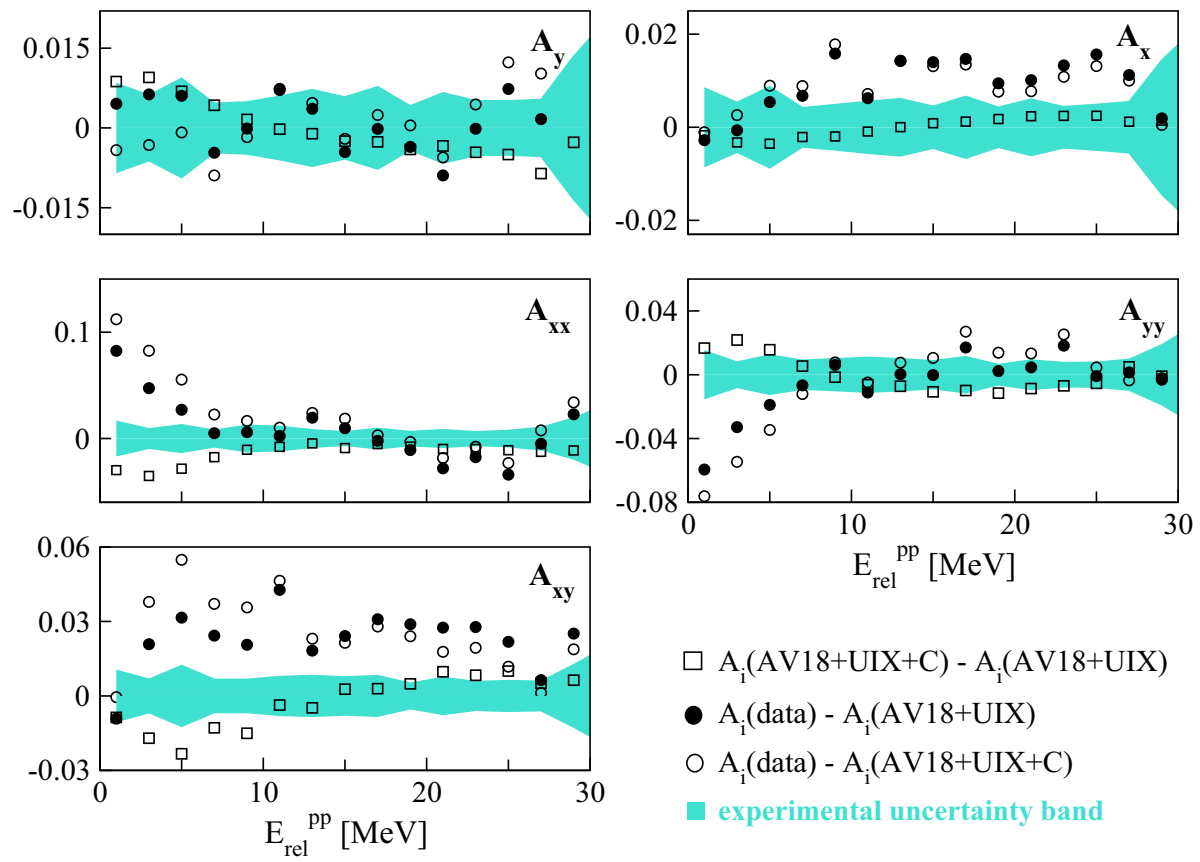

$$
\begin{aligned}
& \square \mathrm{A}_{\mathrm{i}}(\mathrm{AV} 18+\mathrm{UIX}+\mathrm{C})-\mathrm{A}_{\mathrm{i}}(\mathrm{AV} 18+\mathrm{UIX}) \\
& \text { - } \mathrm{A}_{\mathrm{i}}(\text { data })-\mathrm{A}_{\mathrm{i}}(\mathrm{AV} 18+\mathrm{UIX}) \\
& O \mathrm{~A}_{\mathrm{i}}(\text { data })-\mathrm{A}_{\mathrm{i}}(\mathrm{AV} 18+\mathrm{UIX}+\mathrm{C}) \\
& \square \text { experimental uncertainty band }
\end{aligned}
$$

Fig. 2 Similar to Fig. 1, but for the net effects of the Coulomb interaction in vector and tensor analyzing powers. Theoretical calculations by Deltuva et al. For details see text

predictions have been individually subtracted from the data and the results are shown as circles in Fig. 2. The difference between the results of the two types of calculations i.e. the predicted net effects of the Coulomb interaction are shown as squares. In most cases the squares are grouped on the zero level, i.e. the predicted Coulomb effect is negligible. For tensor analyzing powers certain departures from zero are visible at low $E_{\text {rel }}^{p p}$. However, the predicted effects of the Coulomb interaction do not solve but rather exacerbate the problem of describing tensor analysing powers with currently available $2 \mathrm{~N}+3 \mathrm{~N}$ potentials: Clearly, the open circles are even more distant from the zero level than the full ones.

Two-dimensional spectra based on two (out of four) invariants should provide us with deeper insight into the system dynamics. As the first step they are constructed for the differential cross section, which reveals interesting dependencies when studied as a function of $E_{r e l}^{p p}$ alone [11]. The ratio $\frac{\sigma_{2 N+3 N}-\sigma_{2 N}}{\sigma_{2 N}}$ was calculated in order to observe the relative influence of the given $3 \mathrm{NF}$ effect. $\sigma_{2 N+3 N}$ denotes the differential cross section calculated with the $3 \mathrm{NF}$ included, while $\sigma_{2 N}$ are the results of calculations using only the $2 \mathrm{~N}$ potential. The predicted effects of the TM99 $3 \mathrm{NF}$ on the differential cross section of the breakup reaction at $130 \mathrm{MeV}$, presented as a function of $E_{r e l}^{p p}$ and $E_{t r}^{n}$, are shown in Fig. 3, left panel. The analysis has been performed for the region where data measured in the SALAD experiment exist [15], therefore the distributions are shown using the experimental thresholds and acceptance. Colours show changes in the magnitude of the effects. The 3NF effects are very local - the strongest on the very edge of the available phase space at large $E_{r e l}^{p p}$. The experimental data $\sigma_{\text {exp }}$ were compared to the calculations based on the CD-Bonn potential alone and the analogous ratio $\frac{\sigma_{e x p}-\sigma_{2 N}}{\sigma_{2 N}}$ has been constructed. As it is shown in Fig. 3, right panel, the theoretical description based on the $2 \mathrm{~N}$ interaction reveals a deficiency in the same region where $3 \mathrm{NF}$ effects are predicted and of very similar size. There is also an additional region in the data where a departure from the calculations is visible. It is located at the lowest $E_{r e l}^{p p}$ where a strong negative effect can be observed, i.e. the theory underestimates the data, and the effect changes rapidly to a positive one nearby. This region is, as we already know, particularly sensitive to the Coulomb force, which is not included in the calculations presented.

The region sensitive to the Coulomb repulsion can be investigated in detail on the basis of the results of the experiment mentioned earlier, devoted to study Coulomb effects in the forward angular region. The calculations using the AV18 potential and Urbana IX 3NF were used for comparisons, with $\left(\sigma_{2 N+3 N+C}\right)$ and without $\left(\sigma_{2 N+3 N}\right)$ the Coulomb interaction. The theoretically predicted effects $\frac{\sigma_{2 N+3 N+C}-\sigma_{2 N+3 N}}{\sigma_{2 N+3 N}}$ and the comparison of the data with the calculations without Coulomb $\frac{\sigma_{e x p}-\sigma_{2 N+3 N}}{\sigma_{2 N+3 N}}$ are presented in Fig. 4. It becomes 

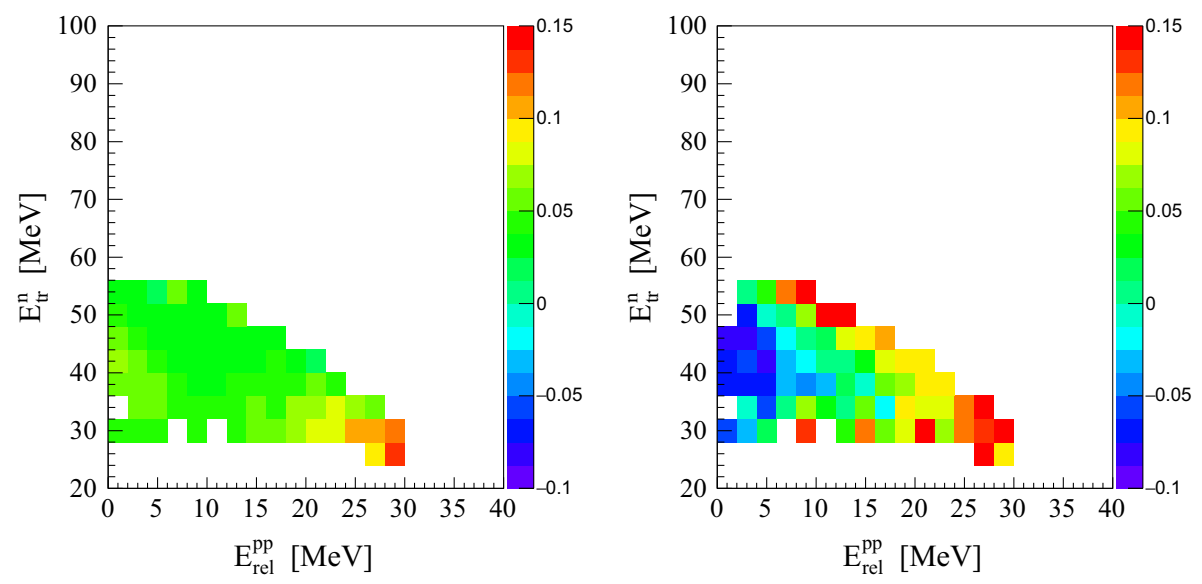

Fig. 3 Net effects of the $3 \mathrm{NF}$ on the differential cross section of the $d p$ breakup at $130 \mathrm{MeV}$, presented as a function of two invariants. Left panel Difference of theoretical predictions (by Witała et al.) obtained for CD-Bonn potential with and without TM99 3NF, relatively normalized to calculations without 3NF. Right panel Difference between experimental data and calculations with CD-Bonn alone, normalized in the same way
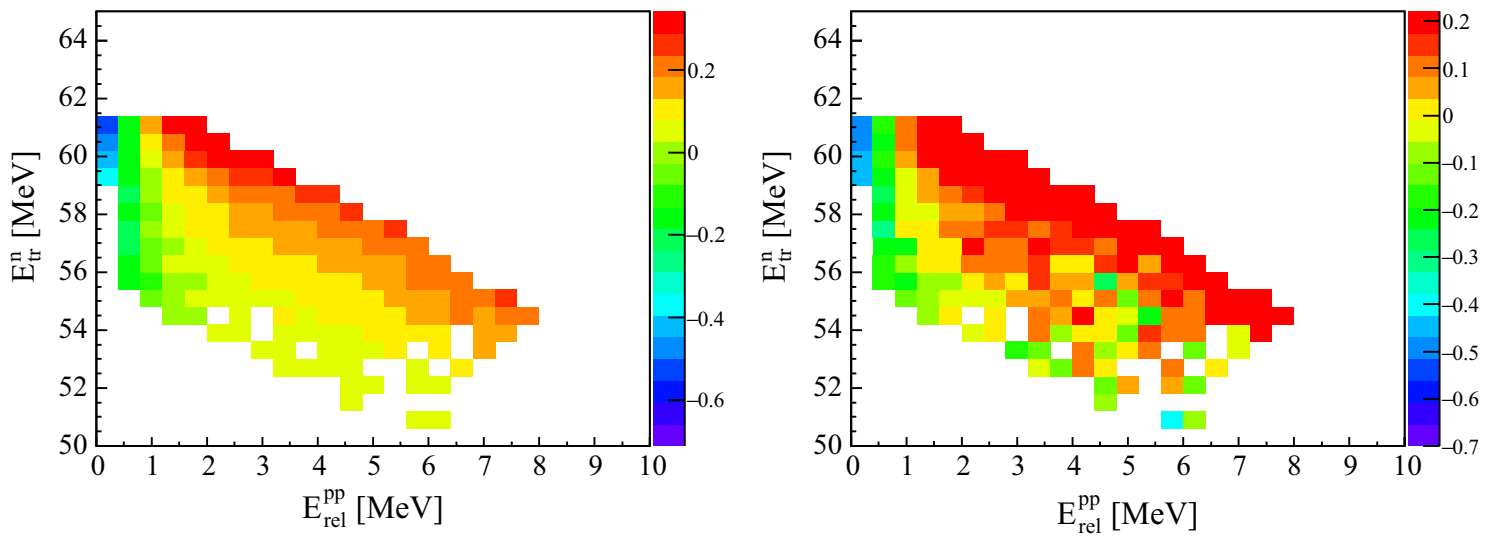

Fig. 4 Similar to Fig. 3, for the net effects of Coulomb interaction in the differential cross section of the $d p$ breakup at $130 \mathrm{MeV}$. Left panel Difference of theoretical predictions (by Deltuva et al.) obtained for AV18 potential and Urbana IX 3NF, with and without Coulomb interaction, relatively normalized to the calculations without the Coulomb interaction. Right panel Difference between experimental data and calculations with AV18 potential and Urbana IX 3NF, normalized in the same way

immediately clear that the angular acceptance of the GeWall detector strongly"zooms in" at a certain region of the $E_{t r}^{n}$ versus $E_{r e l}^{p p}$ plane, probing lower $E_{r e l}^{p p}$ and higher $E_{t r}^{n}$ as compared to the case presented in Fig. 3. In this region, the theoretically predicted influence of the Coulomb force (left panel) changes quite dramatically from negative to positive values, which corresponds to "pushing out" of proton pairs from certain configurations to neighbouring ones. This effect amazingly well reproduces the deficiency of the Coulomb-less calculations in describing the data (right panel).

Extending the studies to the slightly higher deuteron beam energy of $160 \mathrm{MeV}$ ( $80 \mathrm{MeV} /$ nucleon), we have an opportunity to understand how the predicted and measured effects change when investigated in the coordinates proposed here. The experiment at $160 \mathrm{MeV}$ was performed with the use of BINA. The BINA Wall detector represents a very similar angular acceptance, energy thresholds, angular and energy resolution as the SALAD detector used at $130 \mathrm{MeV}$. Therefore the influence of one parameter only, namely of the beam energy, can be studied. Due to the still ongoing analysis we have not shown the comparison to the data yet. The predicted effects are calculated, as in the previous cases, in the kinematic region where the experimental data were measured (excluding the lowest $\phi_{12}$, corresponding to the lowest $E_{r e l}^{p P}$ values). The calculations using the AV18 2N potential with and without the Urbana IX 3NF have been compared. As it might be observed in Fig. 5, an increase of beam energy provides an access to the region of higher $E_{t r}^{n}$ and $E_{r e l}^{p p}$. The predicted 3NF effects are larger than the effects observed at $130 \mathrm{MeV}(65 \mathrm{MeV} /$ nucleon$)$ and cumulated at the highest $E_{\text {rel }}^{p p}$ values. 


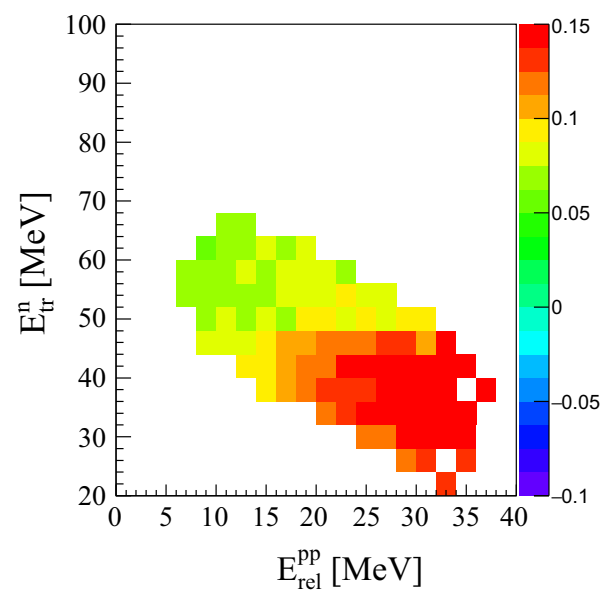

Fig. 5 Similar to Fig. 3, left panel, for the net effects of Urbama IX 3NF studied at the beam energy of $160 \mathrm{MeV}$. Theoretical predictions by Witała et al

\section{Summary}

Due to the recent progress in experimental techniques, the database for 3-nucleon system studies at intermediate energies has been significantly enriched and the progress in this area is continuing. The breakup reaction is studied in experiments employing detection systems covering large parts of the phase space, which allows for systematic analyses of observables as a function of kinematic variables. For the sake of comparing data obtained at different energies and learning more about the dynamics of the process, the analyses should rely on invariants, rather than on the classical kinematic variables related to geometrical configurations. The paper presents a pilot example of that kind of analysis as an indicator of its usefulness. Other combinations of invariants are also being considered and subsequently the analysis will be extended to other beam energies. New data sets will be collected in the near future for the breakup reaction. Thay will complement the basis for the tests of state-of-the-art and forthcoming theoretical calculations, which in turn will help to understand details of the few-nucleon system dynamics.

Open Access This article is distributed under the terms of the Creative Commons Attribution 4.0 International License (http:// creativecommons.org/licenses/by/4.0/), which permits unrestricted use, distribution, and reproduction in any medium, provided you give appropriate credit to the original author(s) and the source, provide a link to the Creative Commons license, and indicate if changes were made.

\section{References}

1. E. Epelbaum, H.W. Hammer, U.G. Meissner, Modern theory of nuclear forces. Rev. Mod. Phys. 81, 1773 (2009)

2. S.A. Coon, H.K. Han, Reworking the Tucson-Melbourne three-nucleon potential. Few Body Syst. 30, 131 (2001)

3. B.S. Pudliner, V.R. Pandharipande, J. Carlson, R.B. Wiringa, Quantum Monte Carlo calculations for $A \leq 6$ nuclei. Phys. Rev. Lett. 74, 4397 (1995)

4. A. Deltuva, R. Machleidt, P.U. Sauer, Realistic two-baryon potential coupling two-nucleon and nucleon- $\Delta$-isobar states: fit and applications to three-nucleon system. Phys. Rev. C 68, 024005 (2003)

5. M. Viviani, A variational approach to three- and four-nucleon systems. Nucl. Phys. A 631, 111c (1998)

6. A. Nogga, H. Kamada, W. Glöckle, Modern nuclear force predictions for the alpha particle. Phys. Rev. Lett. 85, 944 (2000)

7. S.C. Pieper, R.B. Wiringa, Quantum Monte Carlo calculations of light nuclei. Ann. Rev. Nucl. Part. Sci. 51, 53 (2001)

8. P. Navratil, V.G. Gueorguiev, J.P. Vary, W.E. Ormand, A. Nogga, Structure of $A=10-13$ nuclei with two- plus three-nucleon interactions from chiral effective field theory. Phys. Rev. Lett. 99, 042501 (2007)

9. K. Sagara, Experimental investigations of discrepancies in three-nucleon reactions. Few Body Syst. 48, 59 (2010)

10. N. Kalantar-Nayestanaki, E. Epelbaum, J.G. Meschendorp, A. Nogga, Signatures of three-nucleon interactions in few-nucleon systems. Rep. Prog. Phys. 75, 016301 (2012)

11. St Kistryn, E. Stephan, Deuteron-proton breakup at medium energies. J. Phys. G Nucl. Part. Phys. 40, 063101 (2013)

12. A. Ramazani-Moghaddam-Arani et al., Elastic proton-deuteron scattering at intermediate energies. Phys. Rev. C 78, 014006 (2008) 
13. N. Kalantar-Nayestanaki et al., A small-angle large-acceptance detection system for hadrons. Nucl. Instrum. Methods A 444, $591(2000)$

14. St Kistryn et al., Evidence of three-nucleon force effects from $130 \mathrm{MeV}$ deuteron proton breakup cross section measurement. Phys. Rev. C 68, 054004 (2003)

15. St Kistryn et al., Systematic study of three-nucleon force effects in the cross section of the deuteron-proton breakup at 130 MeV. Phys. Rev. C 72, 044006 (2005)

16. E. Stephan et al., Vector and tensor analyzing powers in deuteron-proton breakup at $130 \mathrm{MeV}$. Phys. Rev. C 82, 014003 (2010)

17. M. Eslami-Kalantari et al., Proton-deuteron break-up measurements with BINA at $135 \mathrm{MeV}$. Mod. Phys. Lett. A 24, 839 (2009)

18. H. Mardanpour et al., Spin-isospin selectivity in three-nucleon forces. Phys. Lett. B 687, 14 (2010)

19. H.O. Meyer et al., Axial observables in dp breakup and the three-nucleon force. Phys. Rev. Lett. 93, 112502 (2004)

20. K. Sekiguchi et al., Three-nucleon force effects in the ${ }^{1} \mathrm{H}(\mathrm{d}, \mathrm{pp}) \mathrm{n}$ reaction at $135 \mathrm{MeV} /$ nucleon. Phys. Rev. C 79, 054008 (2009)

21. A. Deltuva, A.C. Fonseca, P.U. Sauer, Momentum-space treatment of the Coulomb interaction in three-nucleon reactions with two protons. Phys. Rev. C 71, 054005 (2005)

22. H. Witała et al., Three-nucleon force in relativistic three-nucleon Faddeev calculations. Phys. Rev. C 83, 044001 (2011)

23. A. Deltuva, Momentum-space calculation of proton-deuteron scattering including Coulomb and irreducible three-nucleon forces. Phys. Rev. C 80, 064002 (2009)

24. St Kistryn et al., Evidence of the Coulomb-force effects in the cross-sections of the deuteron-proton breakup at $130 \mathrm{MeV}$. Phys. Lett. B 641, 23-27 (2006)

25. M. Betigeri et al., The germanium wall of the GEM detector system. Nucl. Instrum. Methods Phys. Res. A 421, 447 (1999)

26. I. Ciepał et al., Investigation of the deuteron breakup on proton target in the forward angular region at $130 \mathrm{MeV}$. Few Body Syst. 56, 665 (2015)

27. H. Witała, J. Golak, R. Skibiński, Selectivity of the nucleon-induced deuteron breakup and relativistic effects. Phys. Lett. B 634, $374(2006)$

28. J. Zejma et al., Cross sections and analyzing powers Ay in the breakup reaction $2 \mathrm{H}(\mathrm{p}, \mathrm{pp}) \mathrm{n}$ at $65 \mathrm{MeV}$ : Star configurations. Phys. Rev. C 55, 42 (1997)

29. M. Allet et al., Proton-induced deuteron breakup at $\mathrm{Elab}^{p}=65 \mathrm{MeV}$ in quasi-free scattering configurations. Few Body Syst. 20, 27 (1996)

30. H. Witała, Private communication

31. B. Kłos et al., Systematic studies of the three-nucleon system dynamics in the cross section of the deuteron-proton breakup reaction. Few Body Syst. 55, 721 (2014)

32. G. Baur, On the Chew-Low plot as a limiting case of the distorted wave theory of break-up reactions. Z. Phys. A 277, 147 (1976)

33. J. Stepaniak, Pion production in 3He-proton interactions. Acta Phys. Pol. B 27, 2971 (1996)

34. I. Ciepał, I. Skwira-Chalot, et al., Applications of polarized deuteron beams for studies of few-nucleon dynamics in d-p breakup, in Proc. of the XVIth International Workshop in Polarized Sources, Targets, and Polarimetry (PSTP2015) PoS 039 